\title{
CAMPESINAS Y DERECHOS HUMANOS: CLAVES PARA ENTENDER LA CIUDADANÍA EN MÉXICO.
}

\section{PEASANT WOMEN AND HUMAN RIGHTS: KEYS TO UNDERSTAND CITIZENSHIP IN MEXICO*}

Adriana Estrada Álvarez ${ }^{1}$

México
Palabras Clave: ciudadanía, derechos humanos, relaciones de poder, esfera pública, feminismo

Keywords: citizenship, human rights, power relations, public sphere, feminism.

\section{RESUMEN}

Este artículo es una reflexión que da claves teóricas para entender los derechos humanos y la ciudadanía de las mujeres campesinas en México. Para ello se ubica al concepto en el marco del desarrollo de la modernidad, el capital y el nacimiento de los Estados-nación. Se desarrolla de manera general las diferentes definiciones que existen en torno a las relaciones de poder y có mo se vinculan con el concepto de ciudadanía. Desde las tradiciones liberal y republicana, y los aportes de la corriente comunitarista, se define la ciudadanía como un proceso vinculado a los conceptos de Estado, democracia, esfera pública y privada, imaginario social, movimiento social y cultura política. Los derechos de las mujeres y la plena realización de su ciudadanía se debaten desde la teoría feminista. Se define el movimiento feminista de manera incluyente, el cual apela a la liberación de la dominación masculina, se describe
* Traducción: Gabriela Guerrero U.

Revisión: Centro de Traducción del Instituto de Idiomas UAm

1 Doctorado en Ciencias Políticas y Sociales de la Universidad Nacional Autónoma de México. El presente artículo es fruto de su investigación doctoral Dirección electrónica de

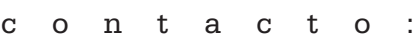
saratrapo@hotmail.com 
como los planteamientos elaborados por las feministas, han trascendido en la concepción original sobre los derechos ciudadanos, y que sólo son realizables a partir de las experiencias de vida concretas de las mujeres. Desde este debate teórico se definen los derechos humanos y la ciudadanía de las mujeres como un proceso histórico donde se disputan relaciones de poder que articula demandas de acuerdo al espacio y tiempo: individual y colectivo; regional, nacional y global.

\section{ABSTRACT}

Abstract: This paper is a reflection which provides theoretical clues to understand human rights and peasant women's citizenship in Mexico. From a conceptual perspective, the concept of citizenship is placed within the framework of the development of modernity, capitalism and birth of nationstates. In general, different existing definitions of citizenship are related to power relationships and how they are linked. From Liberal and Republican traditions, and communitarism's ideas, citizenship is defined as a process linked to the concepts of state, democracy, public and private spheres, social imaginary, social movement and political culture. Women's rights and the fulfillment of their citizenship are debated from a feminist perspective. Feminist movement is defined as inclusive, demanding liberation from male dominance; it is described how the proposals made by feminists have transcended the original perception of citizen's rights, that can only be done based on concrete experiences of women's lives. From this theoretical debate, human rights and women 's citizenship are defined as a historical process where power relations are in conflict, articulating demands according to space and time: individual and collective; regional, national and global.

\section{INTRODUCCIÓN}

El debate sobre la ciudadanía en los últimos años ha cobrado importancia porque se han visibilizado las profundas desigualdades sociales que hacen que algunos seres humanos gocen de pleno ejercicio de sus derechos y otros tengan un acceso precario o inexistente, es decir se construye de manera conflictiva determinada por relaciones de poder económicas, políticas, culturales y sociales.

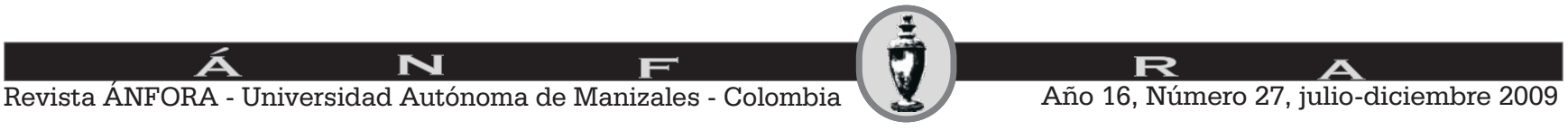


Por ello, el ejercicio pleno de la ciudadanía se debate desde un ideal moral que se configura con el desarrollo de la modernidad, de la economía del capital y el nacimiento de los Estados nacionales, pero en la actualidad cobra un carácter global a nivel de organismos supra-nacionales, como es la Declaración Universal de los Derechos Humanos de la ONU y al cual se apela para legitimar demandas de los grupos sociales subalternos.

El artículo se organiza de la siguiente manera: Primero se desarrolla las diferentes definiciones generales sobre relaciones de poder que existen en el ámbito de la sociología política. Segundo, se desarrolla de manera general la discusión clásica compuesta por la corriente liberal y republicana, principalmente. En el tercer apartado se desarrolla la discusión contemporánea, de manera somera se menciona el debate sobre los derechos sociales y económicos, se introduce la discusión sobre esfera pública como el ámbito donde se construye la ciudadanía, y se vincula con el debate sobre movimiento social, cultura política e imaginario social. Por último se aborda la crítica y los aportes de la teoría y el movimiento feminista.

\section{Diferentes definiciones sobre concepto de relaciones de poder.}

Las relaciones de poder representan el trasfondo de la discusión y ésta tiene diferentes definiciones dentro de la teoría política y sociológica que se vuelve necesario mencionar para poder entender dichos procesos. Como definición básica se entiende como la dominación de un humano por otro humano. (Bobbio, 2002)

Max Weber piensa las relaciones de poder como una relación de mando obediencia que se generan por hábito invertebrado o por coacción y anota:

"...poder significa la probabilidad de imponer la propia voluntad, dentro de una relación social, aun contra toda resistencia y cualquiera que sea el fundamento de esa probabilidad" y más adelante añade que "el concepto de poder es sociológicamente amorfo". (Weber, 1987: 464)

Micheal Foucault piensa el poder como un aspecto de todo ser humano, y en este sentido implica formas de resistencia:

"La dominación es una estructura global de poder cuyas ramificaciones y consecuencias pueden encontrarse a veces hasta en la trama más tenue de la sociedad". (Foucault, 1980: 20)

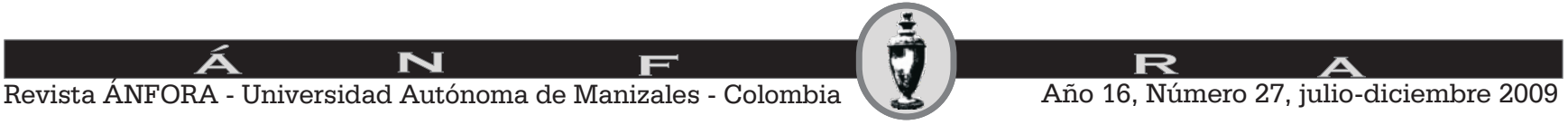


La escuela marxista explica las relaciones de poder a partir de las condiciones materiales y estructuradas de la sociedad. Y Pierre Bourdieu aporta que todo poder implica una dimensión simbólica:

“...debe obtener de los dominados una forma de adhesión que no descansa en la decisión deliberada de una conciencia ilustrada sino en la sumisión inmediata y pre-reflexiva de los cuerpos socializados."

"-El espacio-tiempo doméstico donde la forma de poder es el patriarcado, las relaciones sociales de sexo.

-El espacio-tiempo de la producción, donde el modo de poder es la explotación.

-El espacio-tiempo de la comunidad, donde la forma de poder es la diferenciación desigual, entre quien pertenece a la comunidad y quienes no.

-El espacio-tiempo estructural del mercado, donde la forma de poder es el fetichismo de las mercancías.

-El espacio-tiempo de la ciudadanía, lo que normalmente el espacio público: ahí la forma de poder es la dominación, el hecho de que hay una solidaridad vertical entre los ciudadanos y el Estado.

-El espacio-tiempo mundial en cada sociedad, que está incorporado en cada país, donde la forma de poder es el intercambio desigual." (Souza, 2006:52-53)

Si bien existe una interrelación entre las distintas formas en que se ejerce el poder, de acuerdo al planteamiento de Souza. En esta investigación básicamente se piensa las relaciones de poder desde el espacio-tiempo doméstico y el espacio-tiempo de la ciudadanía.

\section{Las definiciones clásicas de la ciudadanía.}

La ciudadanía como fruto de la reconfiguración de las relaciones de poder que prevalecieron durante el medievo y se cristalizaron con el surgimiento del Estado-moderno (Bendix citado en Luque, 2002: 14), tuvo dos rasgos constitutivos, señalados por el teórico político Jürgen Habermas:

“...la soberanía del poder estatal encarnada en el príncipe y la diferenciación del Estado con respecto a la sociedad, con lo cual se les concedería de modo paternalista a las personas privadas un componente básico de libertades subjetivas. Estos derechos del súbdito se transforman con el cambio de la soberanía del príncipe a la soberanía del pueblo en los derechos del hombre y

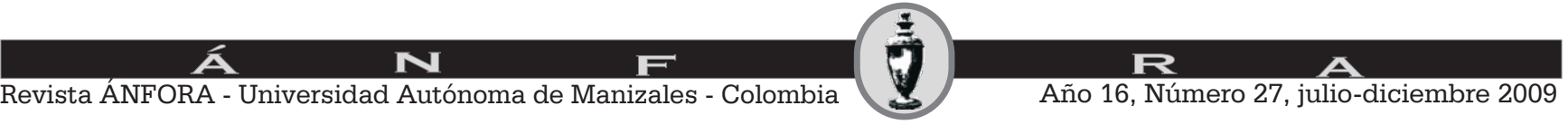


del ciudadano, esto es, en derechos liberales y políticos. Considerado como el tipo ideal, estos derechos garantizan junto a la autonomía privada también la autonomía pública, que en principio es igual para cualquiera." (Habermas, 1999: 89)

El teórico alemán apunta lo siguiente acerca de la importancia de la conformación de los Estados nacionales:

"El mérito del Estado nacional estaba, pues, en que resolvía dos problemas en uno: hizo posible una nueva forma, más abstracta, de integración social sobre la base de un nuevo modo de legitimación." (Habermas, 1999: 38)

La soberanía y el uso legítimo de la violencia (Weber, 2008) son los rasgos principales que definen al Estado moderno. Desde esta definición general se construyen tres corrientes históricas que suceden en distintos tiempos y espacios en el mundo y dan origen a una división política, cultural, social y económica global. T.K. Oomen señala lo siguiente al respecto:

"The bourgeois revolution gave birth to the First Word, the proletarian revolution to the Second and the colonial revolution to the Third. Each of them created expectancies, all of which remain unfulfilled to this day. The liberal expectancies, of the First World postulated the disappearance of primordial ties and traditional loyalties and the emergence of a democratic and rational "man". The radical expectancy of the Second World assumed that "social man" would emerge who would abandon narrow loyalties to the fatherland. The nationalist expectancy of the Third World presumed that the citizens of the new states would waive their particularistic identies and loyalties in favor of building the "nation"'" (Oomen, 1997: 10-11)

Desde la perspectiva racional del primer mundo se construyen dos corrientes históricas, que tienen influencias determinantes en la conformación de los Estados-nación de América Latina: la republicana y la liberal.

De manera muy general la concepción republicana (Europa continental, s. XVIII) se relaciona con las representaciones de poder. El poder del Estado radica en hacer prevalecer el bien común. Apela a la integración social y la participación directa para elegir representantes políticos. La ciudadanía se sustenta en la construcción de una identidad nacional y la libertad se identifica con la esfera pública. (Ochman, 2002: 127)

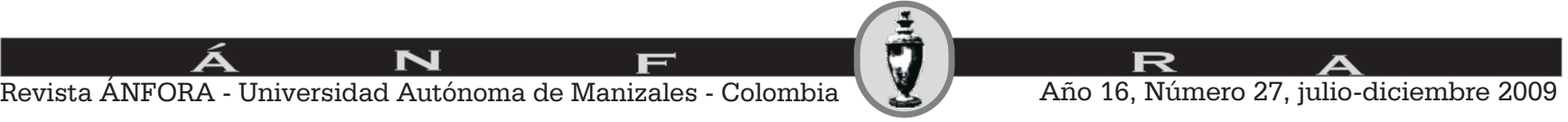


La concepción liberal (Reino Unido, s. XIX) pone énfasis en la garantía de los derechos fundamentales, y limita el poder del Estado a través de la ley y el reconocimiento de los derechos. La ciudadanía se interpreta desde la protección formal de las preferencias individuales y limita lo público. La participación es válida como consentimiento a través de la elección de representantes. La libertad se identifica con la esfera privada. (Ibídem)

Mientras el pensamiento republicano sustenta el reconocimiento de los derechos políticos con base en la existencia de una comunidad política que garantiza la participación política a través del sufragio y el derecho a la representación política del ciudadano, la corriente liberal defiende garantías civiles e individuales que se relacionan con la libertad de expresión, de religión, de propiedad, de realizar contratos y de acceso a la justicia. Estas demandas nacen principalmente de la recién conformada burguesía que busca romper con los antiguos lazos feudales. (Luque, 2002: 15)

Los derechos sociales surgen como respuesta a una serie de demandas de movimientos sociales por la lucha de garantías laborales y condiciones de vida de las clases subalternas (principalmente obreros). También son efecto de un reacomodo de las fuerzas políticas y económicas globales de fin de la posguerra (s.XX). Las cuales dan origen a los llamados países socialistas y los países capitalistas o de primer mundo. Estos últimos optan por la conformación del denominado Estado de Bienestar e implementa políticas donde el Estado es el principal impulsor del desarrollo económico y político de una nación. ${ }^{2}$ El Estado de Bienestar será la corriente que más influencia tiene en América Latina y en especial en México.

\section{La discusión contemporánea sobre ciudadanía y derechos humanos.}

Hacia finales de la década de 1970 inicia un proceso de crisis de desarrollo de los Estado de bienestar, ${ }^{3}$ y se configura un largo reacomodo global de las fuerzas políticas y económicas. Se implementa como forma de desarrollo hegemónico las políticas neoliberales enmarcadas dentro de la globalización. ${ }^{4}$ Proceso que incide de múltiples formas en los pactos fundacionales de los Estado nación.

La ciudadanía es un elemento que vuelve a adquirir importancia en el debate internacional (tanto teórico como a nivel de políticas nacionales y supranacionales), porque se visibilizan las profundas desigualdades que
2 No me interesa abundar en e l t e m a sobre la conformación de la ciudadanía social, ya que no es el elemento central de definición que adopta ésta investigación. Sin embargo, es importante mencionar que T.H. Marshall es el principal teórico que le da sustento a la ciudadanía social, promueve el derecho a la educación y a las garantías laborales económicas como el instrumento de la movilización social. (Luque, 2002: 17)

3 Durante la década de 1970 se dan dos crisis que desestabilizan la economía mundial. La crisis del petróleo y la crisis agroalimentaria. (Rubio, 2000: 80)

4 La globalización es un proceso social, económico, político y cultural, complejo y multidimensional que $\mathrm{t}$ i e $\mathrm{n}$ e $\mathrm{m}$ úl $\mathrm{t}$ i ple s consecuencias entre las que se señala "un aumento de la segregación, separación y marginación social progresiva... " (Bauman, 2004:9)

Una de sus características principales es la transformación de la forma de acumulación y reproducción del capital, el cual pasó de ser productivo a financiero. A esta forma del desarrollo del capitalismo se le denomina excluyente, ya que no incorpora la fuerza de trabajo como parte de la reproducción del capital. (Rubio, 2002) 
persisten, y surgen diferentes críticas que fracturan las visiones tradicionales acerca del ejercicio ciudadano. ${ }^{5}$

Desde la década de 1980 surge un amplio debate en torno al ejercicio ciudadano. Varios temas son los que se disputan, y destaco entre lo que se reconoce como los derechos formales y los derechos realmente existentes.

Los derechos formales representan el orden moral racional al cual aspira el ser humano. Es una construcción teórica, que se compone por el reconocimiento de los derechos políticos y civiles de primera generación; los derechos sociales, económicos y culturales de segunda generación; y durante la década de 1980 se reconocen los derechos de tercera generación que se rigen bajo el principio de la solidaridad, de la cultura de paz, la cooperación, la preservación del medio ambiente y el desarrollo. (Amnistía Internacional)

Una de las características importantes a mencionar dentro del reconocimiento internacional sobre los derechos de primera, segunda y tercera generación es señalado por Saskia Sassen discute desde un análisis de la globalización:

"Los derechos humanos internacionales, en tanto están enunciados en los documentos fundacionales de los Estado nación, son hoy una fuerza que puede socavar la exclusiva autoridad del Estado sobre los ciudadanos... La pertenencia a los Estado-nación deja de ser el único piso para la realización de los derechos... Los derechos humanos comienzan a chocar con el principio de la ciudadanía basada en la nación y las fronteras de la nación." (Sassen, 2003:129)

Elizabeth Jelín vincula el problema de la ciudadanía con las relaciones de poder y define lo siguiente:

“...es una práctica conflictiva vinculada al poder, que refleja luchas acerca de quiénes podrán decir qué en el proceso de definir cuáles son los problemas sociales comunes y cómo serán abordados" (Jelín, 1997:116)

Definición que permite pensar en los derechos realmente existentes a partir de reconocer la ciudadanía como un paradigma universal, pero lo que significa y cómo se experimenta no lo son. Existe una debilidad en el debate teórico en cuanto no se tiene un sustento empírico, que pueda reconocer cuál es la mirada, la perspectiva de las personas, o el imaginario social existente sobre el ejercicio
5 Entre los críticos de la ciudadanía social resaltan Antonin Giddens en la década de 1980

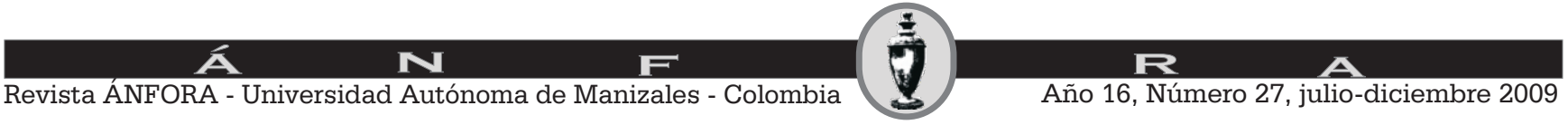


de derechos y el acceso a una ciudadanía plena, donde precisamente existen personas cuyo status de ciudadano no existe o es muy precario. (Kabeer, 2005:1)

Dentro del imaginario social está la forma en que las personas se imaginan su existencia, la relación social entre personas, el cómo se vive y ocurren los actos cotidianos, las esperanzas que se trabajan día con día y el trasfondo en el que descansan dichas expectativas. (Taylor, 2006: 27)

Charles Taylor define que el imaginario social se diferencia de la construcción teórica porque:

“1) se refiere concretamente a la forma en que las personas corrientes "imaginan" su entorno social, algo que la mayoría de las veces no se expresa en términos teóricos, sino que se manifiesta a través de imágenes, historias, leyendas (y yo agregaría experiencia de vida). 2) lo interesante del imaginario social es que lo comparten amplios grupos de personas, si no la sociedad en su conjunto. 3) el imaginario social es la concepción colectiva que hace posibles las prácticas comunes y un sentimiento ampliamente compartido de legitimidad." (Ibídem)

La propuesta de Taylor se articula desde la construcción histórica de larga duración para comprender cómo se configura un pensamiento colectivo con respecto al paradigma de la modernidad, el cual le da legitimidad a lo que entendemos por libertad, igualdad, derechos humanos y democracia. Propuesta que da legitimidad al paradigma de los derechos humanos como el que cobija las diversas nociones sobre lo justo e injusto, y da sentido de identidad colectiva para hacer valer demandas frente al poder político y económico.

Uno de los señalamientos más importantes con respecto a la construcción de ciudadanía y el imaginario social está planteado en la discusión sobre esfera pública y privada, desarrollada principalmente por Habermas, y reconocida por distintos autores como fundamental para poder establecer los lineamentos que permiten entender de mejor manera el tema.

La esfera privada en general se entiende como aquella que se desarrolla en el espacio íntimo que permite la reproducción de la vida social; y la que se establece en términos de mercado, de la apelación del derecho a la propiedad privada de las burguesías

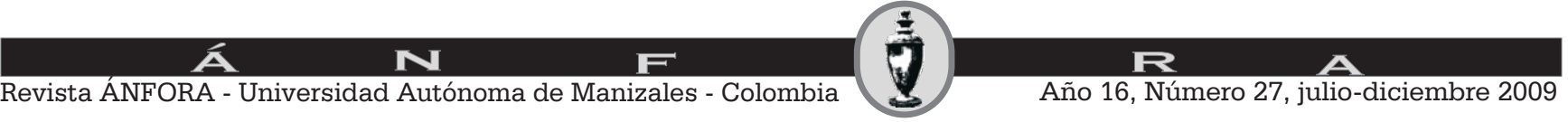


La esfera pública en cambio se define por el espacio donde se construye la legitimidad, autoridad y soberanía del Estado. (PNUD Bolivia, 2007: 35) A través del intercambio de ideas que se establece en el espacio público se configura una opinión en común sin la mediación de la esfera política, ésta última entendida como el espacio de la representación de la toma de decisiones del poder estatal. (Taylor, 2006: 115)

Habermas sostiene que la esfera pública es el espacio donde los problemas privados adquieren una dimensión pública al ser los individuos: mujeres, negros, trabajadores, minorías sociales, etcétera quienes al acceder a ese espacio pueden denunciar una condición de desigualdad. (Habermas citado en Sousa, 2004:47)

Dentro de la esfera pública se produce una acción colectiva de pensamiento común que puede tener implicaciones más complejas, como son los movimientos sociales. (Taylor, 2006: 115)

Desde la teoría sobre nuevos movimientos sociales, Albert Melluci señala que la acción colectiva moderna avanza en dos direcciones: la que se determina a partir de las relaciones de producción; y la acción ciudadana que promueve la realización de los derechos humanos. El sociólogo italiano sostiene que las luchas sociales modernas articulan ambos elementos para configurar movimientos sociales, que define como una acción colectiva que es resultado de una construcción social histórica. (Melluci, 1999: 84)

Esta construcción social histórica tiene como piso una cultura política, entendida como: el espacio y tiempo simbólico en que se reproduce el ejercicio y las estructuras de poder. En ella se expresa una heterogénea y compleja configuración de las relaciones de poder donde están implícitos imaginarios sociales que integran la identidad de un Estado-nacional, y donde los distintos grupos sociales (sean grupos dominantes o clases subalternas) se disputan sus intereses de acuerdo a un ideal a seguir. (Tejera, 2000: 13)

La construcción de ciudadanía y cultura política no siempre confluyen. Puede co-existir una cultura política autoritaria, patrimonial y clientelar que no responda a los ideales bajo los cuales se adscriben los derechos ciudadanos, aunque éstos estén inscritos dentro de la carta constitutiva de un Estado. Es

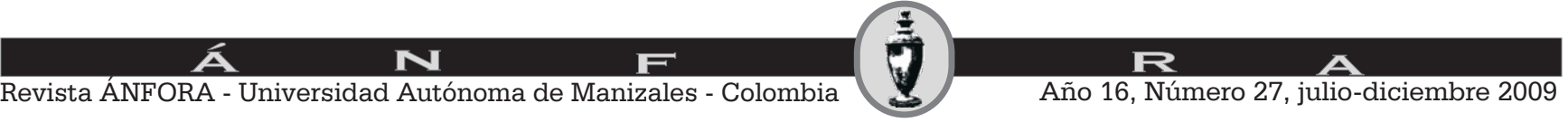


decir, dentro de la cultura política se expresan las múltiples contradicciones donde los derechos humanos y el acceso a la ciudadanía se disputan frente a prácticas que no necesariamente se encaminen al cumplimiento de este ideal.

Esto permite elaborar de manera más compleja el imaginario social bajo el cual se disputa el acceso al ejercicio de una ciudadanía plena, y en particular el reto que significa la plena realización de los derechos de las mujeres.

\subsection{La crítica feminista al concepto de ciudadanía y los derechos humanos de las mujeres.}

La teoría feminista es una de las más críticas frente a las definiciones tradicionales sobre el concepto de ciudadanía. Sostiene que los derechos políticos y civiles se fundaron con una concepción androcéntrica, de la cual las mujeres fueron excluidas de ejercer dichas garantías. Argumenta con respecto a la ciudadanía social que fue concebida desde una perspectiva universal y racional que impidió reconocer las diferencias, conflictos y contradicciones que rigen en cada sociedad. (Mouffe, citado en Bolos, 2008: 35)

La discusión se vincula con un ordenamiento de las relaciones de poder que determinan organización social, política, económica y cultural basada en lo que Pierre Boudiue llama la dominación masculina, y las feministas denominan patriarcado.

La dominación masculina se sostiene a partir de una configuración simbólica que le da estructura a una serie de valores que hacen que las mujeres vivan en una condición subalterna frente a lo masculino. El sociólogo francés lo define de la siguiente forma:

"El cuerpo masculino y el cuerpo femenino, y en especial los órganos sexuales que, como condensan la diferencia entre los sexos, están predispuestos a simbolizarla, son percibidos y construidos según los esquemas prácticos del hábitus y de este modo en apoyos simbólicos privilegiados de aquellos significados y valores que están en concordancia con los principios de la visión falocéntrica del mundo. No es el falo (o su ausencia) lo que constituye el principio generador de esta visión del mundo sino que es esta visión del mundo la que, al estar organizada, por razones sociales que convendrá tratar de descubrir, según la división en géneros

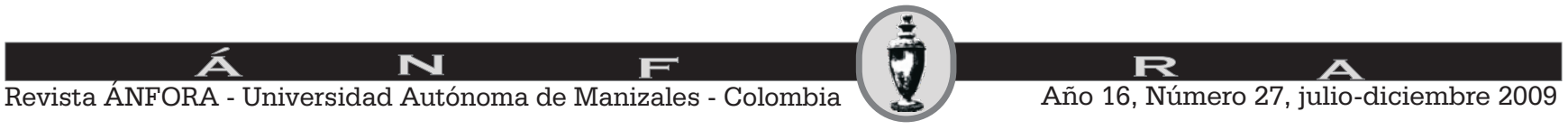


relacionales, masculino y femenino, puede instituir al falo, erigido en símbolo de la virilidad, del nif propiamente masculino, en principio de la diferencia entre los sexos (en el sentido de géneros) y dejar sentada la diferencia social entre dos esencias jerarquizadas en la objetividad de una diferencia natural entre los cuerpos biológicos.

...Lejos de que sean las necesidades de la reproducción biológica los que determinen la organización simbólica de la división sexual del trabajo y, por ende, de todo el orden natural y social, es una construcción arbitraria de lo biológico, y en particular del cuerpo, masculino y femenino, de sus usos y de sus funciones, en especial en la reproducción biológica, que da una base en apariencia natural a la visión masculina de la división del trabajo sexual y de la división sexual del trabajo y, por ende, a toda la visión masculina del mundo." (Bourdieu, 2000: 37)

El ordenamiento de la organización social sustentado en el patriarcado consideró en los inicios de la formación de los Estados-modernos, que las mujeres no eran ciudadanas, ya que el orden natural las hacía ser madres y esposas, confinadas a la esfera privada e íntima porque representaban una masa pre-cívica. (Valcárcel, 2000: 11)

La lucha por los derechos y la ciudadanía de las mujeres se fundamenta en una crítica que busca revertir la relación de dominación masculina, la cual es parte de un proceso histórico que se inicia durante la ilustración (s. XVIII), con la vindicación del reconocimiento de los derechos políticos de las mujeres. En el s. XIX se suma la lucha por los derechos civiles en Inglaterra (declaración de Seneca Falls, 1848). Durante el s. XX se diversifican las luchas y demandas en distintos países y se avanza en el ámbito supra-nacional como nacional. ${ }^{6}$ (Valcárcel, 2000)

Se puede afirmar que la lucha que emprenden las mujeres desde la década de 1970 ha trascendido en los planteamientos originales sobre la concepción de ciudadanía y derechos humanos. Este proceso que en un primer momento se cristaliza con la Primera Conferencia Mundial de la Mujer (México, 1975), visibiliza una condición de desigualdad, discriminación y violencia que viven en los espacios sociales, políticos y culturales; y da pauta para impulsar la Convención sobre la eliminación de todas las formas de discriminación contra la mujer. (Naciones Unidas, 1979)
6 La teoría feminista caracteriza este proceso histórico de lucha por el reconocimiento de los derechos políticos, civiles y sociales de las mujeres en tres olas: La primera ola es la lucha por el reconocimiento de los derechos políticos. La segunda ola es la lucha por los derechos civiles. La tercera ola es la lucha por el reconocimiento de todos los derechos de las mujeres en sus distintas dimensiones. Ver (Valcárcel, 2000) 
En 1985 la Asamblea General de Naciones Unidas declara que toda persona tiene el derecho a la no discriminación por razón de sexo, género, etnicidad, raza, edad, afiliación política, clase social, estado civil, orientación sexual, nacionalidad, religión y discapacidad. Se reconoce la igualdad de acceso de las mujeres al trabajo, salud, educación, y el derecho a la autonomía de las decisiones para las niñas y mujeres. Para ello se determina impulsar políticas y prácticas de reparación, y fomentar la justicia. (Nairobi, 1985)

La Corte Internacional en 1994 reconoce la declaración de 1979 sobre la eliminación de todas las formas de discriminación, desigualdad y violencia contra la mujer (CEDAW, por sus siglas en inglés), y postula una definición legal que pasa a ser normativa para aquellos gobiernos que la suscriben.

Una vez reconocido el sustento político y jurídico internacional, se inicia la conformación de una plataforma de acción (Beijing, 1995) que se sustenta en lo fundamental en promover el pleno ejercicio de los derechos de las mujeres. Esto significa que no basta con declarar el pleno acceso de las mujeres a sus derechos, sino requiere de realizarse en las prácticas que se adoptan, en políticas públicas y en la experiencia de vida de las mujeres.

Generar experiencias de apropiación del derecho como parte fundamental del acceso a una ciudadanía plena, presupone la convicción de legitimidad moral de demandas propias, que se hacen realizables a partir de generar estrategias que pueden expresarse en diálogo, negociación, desencuentros y rupturas (Petchesky, 2006: 61); y en la posibilidad de acceder a los recursos adecuados a nivel institucional que atienda las demandas de la población. Es decir, trascender de las demandas cotidianas a un discurso de justicia social, incursionar en la esfera pública, o en palabras de las feministas que impulsaron el movimiento durante la década de 1970 y 1980 "lo personal es político".

La incursión de las mujeres en la esfera pública es un proceso histórico de lucha, que como se mencionó data del s. XVIII, pero no todas las mujeres incursionaron en este ámbito y el proceso no es homogéneo en el tiempo y el espacio. Es más, existe una crítica al planteamiento sobre la forma de comprender la esfera pública y privada, la cual Seyla Benhabib lo expresa de la siguiente manera:

"Lo que el movimiento de la mujer y las teóricas feministas en las últimas dos décadas han demostrado es que los modos tradicionales de establecer esta

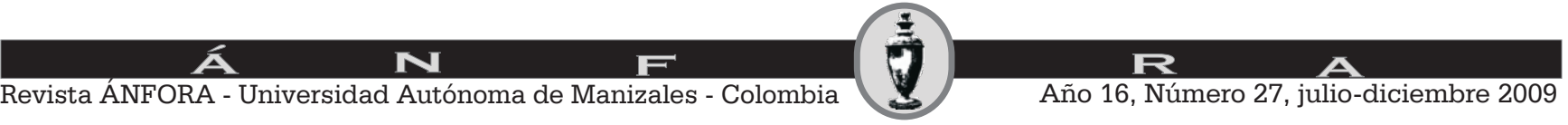


distinción han formado parte de un discurso de dominación que legitima la opresión y la explotación de las mujeres en el ámbito privado. Pero el modelo discursivo, precisamente porque procede a partir de una norma fundamental de reciprocidad igualitaria y porque proyecta la democratización de todas las normas sociales, no puede obviar la democratización de las normas familiares y de las normas que también gobiernan la división por sexos del trabajo en la familia...

La tensión entre la ética discursiva y los modelos de legitimidad y espacio público que se derivan de esto, así como las reivindicaciones del movimiento de la mujer, descansan primordialmente en las fronteras demasiado rígidas que Habermas ha intentado establecer entre las cuestiones de justicia y las de la vida buena, los intereses públicos frente a las necesidades privadas, los valores privados y las normas públicas compartidas...sólo el proceso del discurso sin restricciones y no algún cálculo moral será lo que nos permita restablecer estas fronteras una vez cuestionado su significado tradicional." (Benhabib, 1992: 128-129)

El ideal moral plasmado en los derechos humanos internacionales que incluye la agenda de las mujeres, responde en parte al proceso histórico impulsado desde el movimiento feminista, sin embargo lograr su legitimidad social y cultural responde a procesos más complejos que se vislumbran desde el propio imaginario social que se circunscribe dentro de una cultura política y se cristaliza en las relaciones familiares, locales y regionales de poder, es en ese espacio que pueden ser cuestionados los roles tradicionales e ir delimitando el espacio público y privado.

El movimiento feminista es el principal motor que busca transformar la condición de subordinación que viven las mujeres en el mundo, y que se expresa en múltiples expresiones donde se disputan tendencias diversas acerca de diferentes temáticas que se encuentran en la agenda de los derechos de las mujeres.

Una de las definiciones del movimiento feminista que da cabida a múltiples procesos locales y regionales es la que señala Mouffe (1993):

“...el movimiento feminista da cabida a todos aquellos procesos en los que, mujeres organizadas bajo cualquier modalidad y en cualquier espacio, asumen explícitamente una postura crítica ante las múltiples formas en que

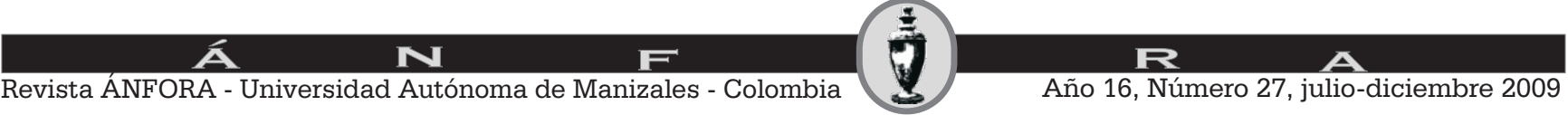


la categoría mujer implica subordinación, injusticia, desigualdad o discriminación, cuestionan las relaciones de poder entre varones y mujeres y se proponen construir formas de relación más igualitarias y libres; sea que se organicen y aboquen exclusivamente a estos objetivos o que articulen sus instancias de mujeres y sus luchas de género a organizaciones mixtas y a reivindicaciones sociales, políticos, ambientales, étnicos, culturales, económicos." (Mouffe, citada en Espinosa, 2008)

Si pensamos el movimiento feminista con esta perspectiva, podemos incursionar en el entendimiento de un abanico de procesos que se construyen desde distintos grupos sociales donde las mujeres incursionan por diferentes motivos en la esfera pública, las cuales actúan desde distintos frentes: privados y públicos, y tienen una postura crítica a la relación de dominación patriarcal.

Esto significa que el paradigma por el que pugna el movimiento feminista ha tenido en principio repercusiones en el imaginario social que se construye y el cual, se puede enriquecer desde realidades muy diversas que no necesariamente responden a una visión europea u occidentalizada de los derechos de las mujeres. Esta definición permite pensar en conciliar ciertos temas que son punto de disputa dentro del movimiento feminista, como son el tema del reconocimiento que cruza la discusión sobre los derechos humanos de las mujeres y el acceso a una ciudadanía plena, y el cual tiene distintas aristas. En este artículo sólo se reflexiona sobre el problema del reconocimiento en el ámbito de la construcción de la ciudadanía.

En la teoría feminista y su movimiento se plantea la disyuntiva entre el reconocimiento de la igualdad y el de la diferencia, la cual ha llevado a un debate al parecer irreconciliable, existe una corriente de investigadoras que piensa que es una discusión que se complementa.

La lucha por el reconocimiento de la igualdad se resume a partir de la exclusión de las mujeres a la ciudadanía. La cual apela a la inclusión de las mujeres dentro del proyecto de la modernidad y su incorporación en todos los ámbitos de la vida social y política. (Amorós citado en Espinoza, 1994)

El feminismo de la diferencia por el contrario piensa que hay que establecer un paradigma diferente al planteado por la modernidad, porque al estar

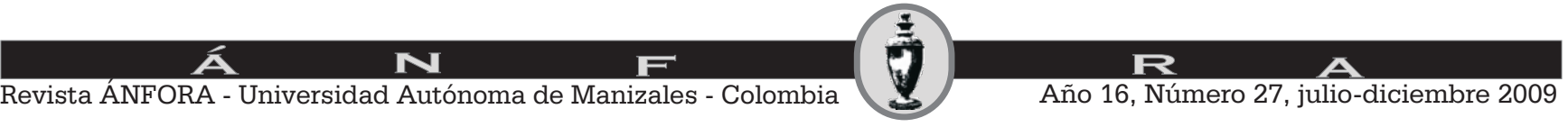


fundamentado en el patriarcado, al entrar en la lucha por el poder tiene implícito:

"Corromperse, entrar en competencia, confirmar el consabido mujer/mal, entrar en lo hombre." (Valcárcel, 1991:55)

Más allá de satanizar el problema de la lucha por el poder, una cuestión que parece fundamental que aporta el feminismo de la diferencia, es que se reconoce una condición diferenciada con respecto a la condición de los varones. Esto permite coincidir con Gutiérrez que cuestiona dicha disyuntiva:

“...reivindicar la igualdad a toda costa sin dar espacio a la diferencia, o reivindicar la diferencia y la particularidad sin la posibilidad de recuperar algún criterio de igualdad... Estos conceptos no son opuestos, la oposición se halla entre igualdad y desigualdad; y si de lo que se trata es de desarraigar las diversas formas en que la categoría mujer es subordinada, sojuzgada, discriminada, se puede perfectamente luchar por la igualdad y a la vez por la diferencia. ¿Acaso las legislaciones laborales especiales para mujeres embarazadas son injustas? Aquí, reivindicar la diferencia sería lo correcto. ¿Acaso es justo que mujeres con liderazgo y capacidad técnica o política sean discriminadas de puestos directivos simplemente por ser mujeres? Aquí lo que procede es la aplicación del criterio de igualdad." (Gutiérrez citada en Espinoza, 2008)

En el contexto de la lucha por la igualdad y marcar las diferencias entre hombres y mujeres, se encuentra también el debate sobre la reproducción de los valores patriarcales por parte de las mujeres desde su condición subalterna, el cual requiere de reconocerse en la búsqueda de construir ciudadanía y trascender su opresión.

La afirmación de los derechos de las mujeres pasa por un proceso de autoreconocimiento que las feministas han denominado como empoderamiento. Concepto que se acuña dentro del movimiento feminista estadounidense (empowerment), que se define como potenciar o tomar el poder sobre. K. Young aporta una definición integral:

“...comprende la alteración radical de los procesos y estructuras que reproducen la posición subordinada de las mujeres como género. En otras palabras, las estrategias para el empoderamiento no pueden ser sacadas de su contexto histórico, que creó la carencia de poder en primer lugar, como

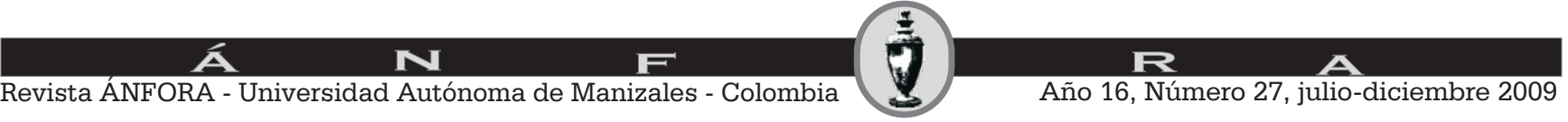


tampoco pueden ser vistas aisladamente de los procesos presentes." (Young, 1997: 105)

El empoderamiento no es un fin en sí mismo, sino una condición que permite trascender hacia la afirmación de derechos desde una perspectiva más compleja que es realizable a partir del: reconocimiento-conocimiento de una condición de género; de una superación de la autoestima; de generar la independencia económica; y de incursionar en lo público y transformar las relaciones privadas con el fin de trascender la condición subalterna, es decir, la configuración de autonomía.

Desde esta perspectiva, podemos pensar que la afirmación de los derechos de las mujeres, es una cuestión que se establece a partir de la configuración de una esfera pública donde el tema a compartir se desarrolla a partir de experiencias y expectativas de vida que permiten caminar hacia una construcción de un discurso afirmativo que incide en el ámbito de las relaciones que se establecen entre hombres y mujeres, tanto en el nivel público como privado.

Se desdibuja la diferencia entre lo público y lo privado, en el sentido que las necesidades pueden nacer de lo privado y trascender en demandas políticas, o demandas políticas trascender en acciones en el espacio privado. Proceso que puede abrir múltiples caminos hacia la negociación, que se desarrolla desde experiencias subjetivas, que se configuran en el imaginario social, y donde se pueden establecer los límites rompiendo con las visiones tradicionales, como lo señala Benhabib.

Una cuestión que señala Young es la necesidad de contemplar el contexto histórico y presente de la condición específica que enfrentan las mujeres, la cual me permito articular con el planteamiento epistemológico que desarrolla Souza en su propuesta de sociología de las ausencias que distingue cinco ecologías: ${ }^{7}$ la de los saberes, que se define como el uso contra hegemónico de la ciencia hegemónica; las temporalidades, el reconocimiento de la existencia de otros tiempos y espacios; reconocimiento de las diferencias a partir de eliminar las jerarquías; trans-escala que se refiere a la relación y trascendencia entre lo local-nacional-global; y el de las productividades, la recuperación de los sistemas alternativos de producción.
7 Souza desarrolla todo un $\mathrm{p}$ l a $\mathrm{n}$ t e a $\mathrm{m}$ i e $\mathrm{n}$ t o epistemológico sobre el abordaje del conocimiento y propone el desarrollo de la sociología de las ausencias, la cual se caracteriza por:

"hacer que lo que está ausente esté presente, que las experiencias que ya existen pero son invisibles o no creíbles estén disponibles; o sea, transformar los objetos ausentes en objetos presentes. Nuestra sociología no está preparada para esto, nosotros no sabemos trabajar con objetos ausentes, trabajamos con objetes presentes; esa es la herencia del positivismo. Estoy proponiendo, pues, una sociología insurgente.

$\mathrm{Si}$ es así, esta falta, esta ausencia, es un desperdicio de experiencia. La manera en que procede la Sociología de las Ausencias es sustituir las monoculturas por las ecologías, y lo que les propongo son cinco ecologías, donde podemos invertir esta situación y crear la posibilidad de que estas experiencias ausentes se vuelvan presentes..." (Souza, 2006:26)

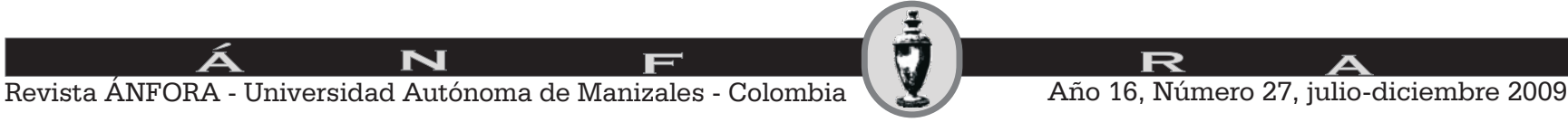


Por el momento me interesa resaltar la ecología de la temporalidad. La afirmación de derechos no sucede en el tiempo y espacio de manera lineal, por el contrario, sucede de manera asincrónica que posibilita pensar que en el tiempo lineal existen diferentes temporalidades, que en un mismo espacio se toman acciones y posiciones diferentes.

Si esto se traduce a la configuración de prácticas de afirmación de derechos, encontramos que no es el mismo tiempo y espacio para mujeres de ciudades que para mujeres de regiones campesinas, mujeres del norte que del sur, de una comunidad a otra, de una generación a otra, donde son múltiples los factores que determinan dicho proceso, marcados por una historia, por valores culturales, por experiencias y necesidades cotidianas, que se desarrollan con ritmos diferentes.

Esto significa, que el punto de partida para acceder a una plena realización de los derechos, no es el mismo para todas las mujeres, y que su realización sólo puede ser a partir de contemplar temporalidad y autonomía, es decir:

“...servirse de su propio entendimiento sin dirección ajena”. (Gilly, 2005)

\section{CONCLUSIÓN}

En resumen, para poder hablar de procesos de afirmación de derechos y construcción de ciudadanía se requiere contemplar lo siguiente:

1.El ejercicio de los derechos humanos y la ciudadanía se desarrolla como procesos que se construyen de manera conflictiva marcadas por las relaciones de poder, en el marco de una historicidad que da origen a la conformación de la modernidad, de los Estado-nación y de la democracia.

2. Tanto la teoría como el movimiento feminista representan una corriente crítica de las formas tradicionales de comprender los derechos humanos y la ciudadanía. Por ello, existe una elaboración histórica que permite actualmente pensar en la plena realización de ésta desde un ideal moral planteado en el ámbito internacional.

3. Su realización depende de la legitimidad que adquiere en las experiencias y realidades concretas, a pesar de que existe un planteamiento moral que resume los derechos formales de las mujeres.

4. Esto lleva a reconocer cómo se negocian sus derechos en la casa, en la

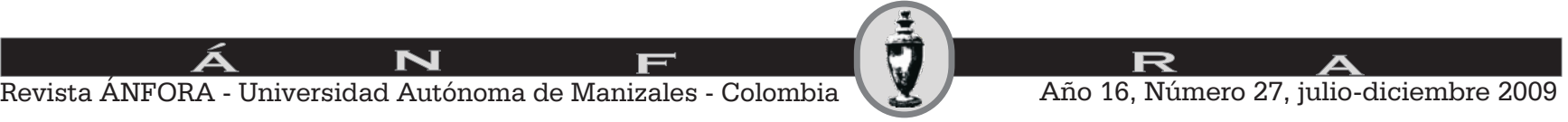


organización, en el trabajo, es decir, en todos los ámbitos de sus relaciones dirigidos a conformarse como sujetos autónomos.

5. Y por último, tanto los derechos humanos de las mujeres como el acceso a una ciudadanía se construye de acuerdo a los contextos sociales, económicos, políticos y culturales específicos. Lo que permite comprender que existen diferentes espacios y tiempos, y que demandas que adquieren sentido para algunas no necesariamente sea el tiempo y el espacio para otras.

\section{BIBLIOGRAFÍA}

Amorós, Celia. (1994) Feminismo, igualdad y diferencia. UNAM-PUEG, México.

Benhabib, Seyla. (2006) El Ser y el Otro en la ética contemporánea. Feminismo, comunitarismo y posmodernismo. Ed. Gedisa. España.

Bolos, Silvia. (2008) Mujeres y Espacio Público. Construcción y ejercicio de Ciudadanía. Ed. Universidad Iberoamericana. México.

Bourdieu, Pierre. (2000, $5^{\text {a }}$ ed. 2007) La dominación masculina. Ed. ANAGRAMA. Barcelona

Espinoza Damián, Gisela (2008) Cuatro Vertientes del Feminismo en México. Diversidad de rutas y cruce de caminos. En Prensa.

Espinosa Gómez, Rosa Aurora. (2004) Expresiones de liderazgo, poder y desarrollo rural en dos grupos de mujeres en el Bajío Guanajuatense. Tesis de Doctorado en Antropología. UNAM. México

Foucault, Michel (1980): Power/Knowledge: Selected Interviews \& Other Writings, 1972-1977. Ed. Colin Gordon. Trans. C. Gordon et al. New York: Pantheon Books. USA.

Gilly Adolfo (2005) Historia a contrapelo una constelación. Era. México.

Habermas, Jürgen. (1999) La inclusión del otro. Ed. Paidos. España.

Jelín, Elizabeth. (2004) Ciudadanía, derechos e identidad. En From the

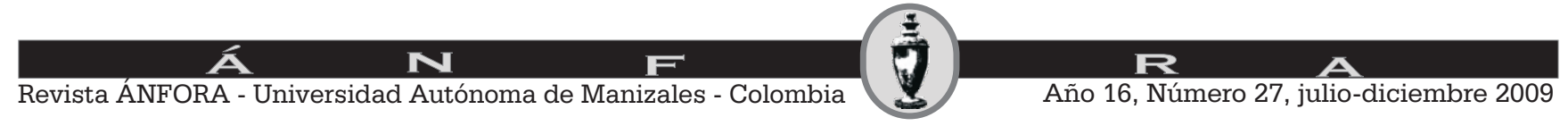


marginality of the 1960 's to the "new poverty" of toyday: a LARR research forum.

Jelín, Elizabeth. (1997) "La construcción de la ciudadanía: entre la solidaridad y la responsabilidad", en Jelín, Elizabeth y Eric Hershberg (coord), Construir la democracia: derechos humanos, ciudadanía y sociedad en América latina, Nueva Sociedad. Venezuela.

Kabeer, Naila. (2005) Inclusive Citizenship. Meanings and Expressions. Ed. Zed Books. London and New York.

Luque, José. Los caminos de la ciudadanía. Tesis para optar el título de Maestro en Ciencias Sociales. Flacso, México.

Mellucci, Alberto. (2002) Acción colectiva, vida cotidiana y democracia. Ed. COLMEX. México.

Ochman, Marta. (2002) La ciudadanía en México: debate teórico. Ed. ITESM. México.

Oommen, T.K. (1997) Citizenship, Nationality and Ethnicity. Politic Press. UK.

Petchesky Rosalinda P. y Karen Judd. (2006) Cómo negocian las mujeres sus derechos en el mundo. Una intersección entre culturas, política y religiones. COLMEX, México.

Sassen, Saskia. (2003) Los espectros de la globalización. FCE. Argentina.

Sousa Santos, Boaventura. (2006) Renovar la teoría crítica y reinventar la emancipación social. (encuentros en Buenos Aires). Argentina.

Taylor, Charles. (2006) Imaginarios sociales modernos. Ed. Paidos. España

Válcarcel, Amelia. (1991) Los desafíos del feminismo ante el s. XXI. Ed. Instituto Andaluz de la Mujer. España

Weber, Max. (XVII ed. 2008) Economía y Sociedad. FCE. México.

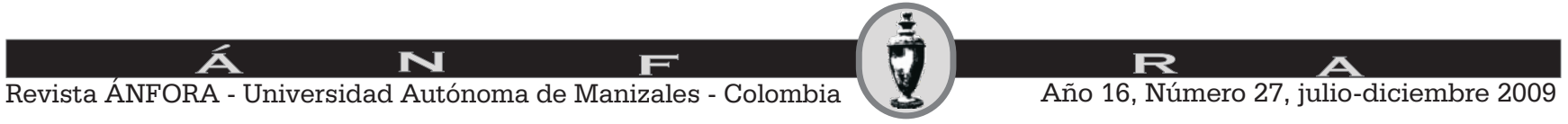


Bibliografía que proviene de Organismos Institucionales.

Amnistía Internacional. La Declaración Universal de los Derechos Humanos. Los derechos de tercera generación.

http://www2.amnistiacatalunya.org/edu/es/historia/dh-futuros.html

Organización de Naciones Unidas. (1985) Declaración de Beinging.

http://www.feministasbeijing10.org.uy/02decla001.htm

Organización de Naciones Unidas. (2007) Declaración de Nairobi sobre el derecho de las mujeres y las niñas a interponer recursos y obtener reparaciones. Nairobi, Kenia, del 19 al 21 de marzo del 2007.

Organización de Naciones Unidas. (1975) Primera Conferencia Mundial sobre la Mujer. México.

Organización Panamericana de la Salud para la Organización Mundial de la Salud. (2002) Informa mundial sobre la violencia y la salud. Washington D. C. USA. 